\title{
Localized and periodic wave patterns for a nonic nonlinear
}

\section{Schrödinger equation}

Kwok W. Chow ${ }^{*}$ and Colin Rogers*

\# (corresponding author) Department of Mechanical Engineering

University of Hong Kong, Pokfulam, Hong Kong

Email: kwchow@hku.hkＰhone: (852) 28592641 Fax: (852) 28585415

* Australian Research Council Centre of Excellence for

Mathematics \& Statistics of Complex Systems;

School of Mathematics and Statistics,

University of New South Wales, Sydney, NSW 2052, Australia

PACS: 05.45.Yv; 42.25.Bs; 42.65.Wi; 02.30.Jr

Re-submission date: April 2013

Physics Letters A: Nonlinear Science

PLA-D-13-00185 


\begin{abstract}
Propagating modes in a class of 'nonic' derivative nonlinear Schrödinger equations incorporating ninth order nonlinearity are investigated by application of two key invariants of motion. A nonlinear equation for the squared wave amplitude is derived thereby which allows the exact representation of periodic patterns as well as localized bright and dark pulses in terms of elliptic and their classical hyperbolic limits. These modes represent a balance among cubic, quintic and nonic nonlinearities.
\end{abstract}

Keywords: Nonlinear Schrödinger equations; ninth order nonlinearity. 


\section{Introduction}

Nonlinear Schrödinger (NLS) equations with cubic and quintic nonlinearities have been investigated extensively both in a theoretical context and in many applied disciplines, notably in hydrodynamics, nonlinear optics and the study of Bose-Einstein condensates (see e.g. [1 - 8]). Here, a class of propagating wave patterns is investigated for families of derivative NLS equations which incorporate seventh (septic) and ninth order (nonic) nonlinearities in addition to those of the standard cubic and quintic kinds. In the setting of optical waveguides, such power law nonlinearities can be interpreted as higher order corrections to the conventional Kerr nonlinearity.

Solitary pulses for a NLS equation with just one nonlinearity of an arbitrary odd power have been analyzed in [9]. For NLS models with nonlinear damping and saturable amplification, the effects of perturbations on the variation of the soliton parameters to leading order have been calculated in [10]. Such considerations can be extended to a 'dual-power' case where two odd order nonlinear terms are present [11].

Importantly, disintegration or blow-up phenomena can occur in NLS equations which incorporate a single nonlinear term representing septic, nonic or higher order effects [12]. Dark solitons can arise in models incorporating self- 
steepening and third order dispersion [13]. Phase plane analysis of solitons, kinks and periodic patterns has been conducted in [14].

Self-steepening and self-frequency shift effects occur commonly in the physics of optical waveguides, and are predicted theoretically by derivative nonlinear Schrödinger (DNLS) equations $[15,16]$. DNLS models with quintic terms have been adopted in the analysis of ultrashort solitons in metamaterials [17].

Here, our concern is with a broad class of DNLS equations which admits both seventh and ninth order nonlinearities as special cases. A procedure recently employed in [18-20] based on the application of a pair of invariants of motion is applied to this DNLS class. A nonlinear structural equation for a squared wave amplitude is derived which can be linked to classical elliptic integrals. This allows the construction of a variety of exact propagating wave patterns for the class of DNLS equations under investigation. In particular, a 'bright' localized solitary pulse with intensity varying as the square root of hyperbolic secant is derived in a long wave limit. In addition, a family of periodic patterns is expressed in terms of a rational expression of the Jacobi elliptic function dn. In the long wave limit, a ‘dark’ pulse is recovered. Modulation instability aspects of the background plane wave are commented upon. Finally, the consideration is extended to general classes of DNLS equations with polynomial nonlinearities, and in particular to nonic DNLS equations. 


\section{The nonic derivative nonlinear Schrödinger equation: Application of a pair of invariants in an integrable subsystem}

Here, we consider the evolution of a slowly varying envelope $A$ as modeled by a derivative nonlinear Schrödinger equation

$i A_{t}+\lambda A_{x x}+\mu|A|^{2} A+i \alpha|A|^{2} A_{x}+v|A|^{4} A+\delta|A|^{6} A+\sigma|A|^{8} A=0$,

incorporating, at the highest order, a nonic nonlinearity. In the setting of optical fiber waveguides, terms involving $\lambda, \mu$ and $\alpha$ are associated with group velocity dispersion, Kerr (cubic) nonlinearity, and self-steepening (or more precisely, an effect which may be converted to self-steepening form following a gauge transformation). The higher order amplitude terms are needed for sufficiently short pulses. The coordinates $t$ and $x$ denote propagation distance and retarded time in temporal waveguides, but represent transverse distance and diffraction in spatial waveguides.

The procedure presented here in fact applies to integrable subsystems of more general NLS equations of the type [20]

$$
i A_{t}+A_{x x}+h^{\prime}(\Sigma) A+j^{\prime}(\Sigma) i A_{x}+(g(\Sigma))_{x} i A-\frac{s|A|_{x x}}{|A|} A=0,
$$

where $\Sigma=|A|^{2}$ and $h, j, g$ are arbitrary analytic functions of $\Sigma$. A prime indicates a derivative with respect to this squared amplitude $\Sigma$. 
If the wave packet ansatz

$$
A=[\phi(x-c t)+i \psi(x-c t)] \exp [i(p x-\Omega t)]
$$

is introduced into (1a), then a coupled nonlinear system results, namely

$$
\begin{aligned}
& \lambda \ddot{\phi}+\left[c-2 \lambda p-\alpha\left(\phi^{2}+\psi^{2}\right)\right] \dot{\psi}+\left[\Omega-\lambda p^{2}+(\mu-\alpha p)\left(\phi^{2}+\psi^{2}\right)\right. \\
& \left.\quad+v\left(\phi^{2}+\psi^{2}\right)^{2}+\delta\left(\phi^{2}+\psi^{2}\right)^{3}+\sigma\left(\phi^{2}+\psi^{2}\right)^{4}\right] \phi=0, \\
& \lambda \ddot{\psi}-\left[c-2 \lambda p-\alpha\left(\phi^{2}+\psi^{2}\right)\right] \dot{\phi}+\left[\Omega-\lambda p^{2}+(\mu-\alpha p)\left(\phi^{2}+\psi^{2}\right)\right. \\
& \left.\quad+v\left(\phi^{2}+\psi^{2}\right)^{2}+\delta\left(\phi^{2}+\psi^{2}\right)^{3}+\sigma\left(\phi^{2}+\psi^{2}\right)^{4}\right] \psi=0
\end{aligned}
$$

where the dot indicates a derivative with respect to the argument $x-c t$. It will be demonstrated that the system (3) admits two independent integrals of motion and, accordingly, is integrable. Thus, it is readily seen that the nonlinear coupled system admits the constant of motion $J$ given by

$$
J=\psi \dot{\phi}-\phi \dot{\psi}+\left(\frac{c}{2 \lambda}-p\right) \sum-\frac{\alpha}{4 \lambda} \sum^{2} .
$$

For the generalized system (1b), the corresponding constant of motion is given by

$$
J=\psi \dot{\phi}-\phi \dot{\psi}-\Pi\left(\sum\right)
$$

where

$$
\Pi\left(\sum\right)=j\left(\sum\right) / 2+\int g^{\prime}\left(\sum\right) \sum d \sum+(p-c / 2) \sum,
$$

where again $\sum=\phi^{2}+\psi^{2}$ is the squared wave packet amplitude. The identification of constants of motion has been proven as key to the subsequent integration of 
other physically important nonlinear dynamical systems, such as the ErmakovRay-Reid systems [21 - 25]. Such systems admit an invariant of the form $I=(\psi \dot{\phi}-\phi \dot{\psi})^{2}-\mathcal{E}(\phi / \psi)$ where $\varepsilon$ denotes an arbitrary analytic function. These Ermakov-Ray-Reid systems have extensive applications in physics, notably in nonlinear optics (see [26, 27] and work cited therein). In recent work, overarching nonlinear integrable systems admitting pairs of invariants which include (1b) and the standard Ermakov-RayReid system have been constructed [28].

Here, the present system (3) admits a second constant of motion

$$
H=\frac{1}{2}\left[\dot{\phi}^{2}+\dot{\psi}^{2}+\left(\frac{\Omega}{\lambda}-p^{2}\right) \sum+\left(\frac{\mu-\alpha p}{2 \lambda}\right) \Sigma^{2}+\frac{v \Sigma^{3}}{3 \lambda}+\frac{\delta \Sigma^{4}}{4 \lambda}+\frac{\sigma \Sigma^{5}}{5 \lambda}\right]
$$

where $H$ is a Hamiltonian. The corresponding Hamiltonian for the generalized system (1b) is

$$
H=\frac{1}{2}\left[\dot{\phi}^{2}+\dot{\psi}^{2}+h(\Sigma)-p j(\Sigma)+\left(\Omega-p^{2}\right) \sum-2 \int g^{\prime}\left(\sum\right)[\Pi(\Sigma)+J] d \sum-\frac{s}{4} \frac{\dot{\Sigma}^{2}}{\Sigma}\right] .
$$

The identity

$\left(\phi^{2}+\psi^{2}\right)\left(\dot{\phi}^{2}+\dot{\psi}^{2}\right)-(\phi \dot{\phi}+\psi \dot{\psi})^{2} \equiv(\phi \dot{\psi}-\psi \dot{\phi})^{2}$

combined with the two integrals of motion (4a), (5a) shows that the squared modulus $\Sigma$ of the envelope is determined by the nonlinear equation 


$$
\begin{aligned}
\dot{\Sigma}^{2} & =4 \sum\left\{2 H+\left(p^{2}-\frac{\Omega}{\lambda}\right) \sum+\left(\frac{\alpha p-\mu}{2 \lambda}\right) \sum^{2}-\frac{v \sum^{3}}{3 \lambda}-\frac{\delta \sum^{4}}{4 \lambda}-\frac{\sigma \sum^{5}}{5 \lambda}\right\} \\
& -4\left[J+\left(p-\frac{c}{2 \lambda}\right) \sum+\frac{\alpha \sum^{2}}{4 \lambda}\right]^{2} .
\end{aligned}
$$

A corresponding expression for a special version of the generalized system (1b) will be given in Section 5 .

For system (1a), to recover the corresponding complex envelope $A$ from $\Sigma$, auxiliary variables $\Theta$ and $\Delta$ are introduced given by

$$
\Theta=\tan ^{-1} \Delta=\tan ^{-1}\left(\frac{\phi}{\psi}\right), \quad \Delta=\frac{\phi}{\psi}
$$

whence

$\Theta=\int^{x-c t} \frac{J+\left(p-\frac{c}{2 \lambda}\right) \sum+\left(\frac{\alpha}{4 \lambda}\right) \Sigma^{2}}{\Sigma} d \xi$,

where $\xi$ is a dummy variable of integration. The resulting exact solution of the envelope equation (1a) is then given by

$A=i \Sigma^{1 / 2} \exp [-i \Theta+i(p x-\Omega t)]$.

It should be remarked that the constants $I, J$ in the reduction procedure above typically arise out of translation of time / space, and gauge invariance for the governing NLS equations. 
The above procedure will now be employed to formulate an algorithm to obtain exact propagating modes in terms of elliptic functions. Two approaches will be adopted. Firstly, elementary transformations are employed to generate expressions related to elliptic functions which solve higher order (including nonic) DNLS equations. Secondly, we identify higher order 'self-steepening' nonlinearities which can be solved by elliptic functions, i.e. integrals involving the reciprocal of fourth order polynomials.

\section{A bright solitary pulse}

The solution procedure now involves the alignment of the equation for the squared modulus (6) with canonical nonlinear equations associated with the classical elliptic functions such as the Jacobi sn, cn, dn functions. As an initial illustration, a localized pulse on a zero background (termed 'bright' in the optical context) is presented. Here, we select alignment with the nonlinear equation

$\left(\frac{d y}{d x}\right)^{2}+1-k^{2}+\left(k^{2}-2\right) y^{2}+y^{4}=0$,

where $k$ is the modulus of the Jacobi elliptic function. This admits a particular solution $y=\operatorname{dn} x$. The substitution $y=u^{2} / A_{0}^{4}$ in the long wave limit $k \rightarrow 1$ takes (10) to 
$\left(\frac{d u}{d x}\right)^{2}-\frac{u^{2}}{4}+\frac{u^{6}}{4 A_{0}^{8}}=0$

with a solution

$u=A_{0}^{2}[\operatorname{sech}(x)]^{1 / 2}$.

On alignment of (6) and (11) it is seen that the envelope equation (6) admits a special solution

$\Sigma=A_{0}^{2} \operatorname{sech}^{1 / 2}(x-c t)$

where the constants of motion $J$ and $H$ both vanish while the amplitude parameter $A_{0}$, angular frequency $\Omega$, and phase speed $c$ are given, in turn, by

$A_{0}=\left(\frac{5 \lambda}{16 \sigma}\right)^{1 / 8}, \quad \frac{\Omega}{\lambda}=\frac{c}{2 \lambda}\left(2 p-\frac{c}{2 \lambda}\right)-\frac{1}{16}, \quad c=\frac{2 \lambda \mu}{\alpha}$,

while

$\delta=0, \quad v=-\frac{3 \alpha^{2}}{16 \lambda}$

The phase variable of the slowly varying envelope is given by

$\Theta=\left(p-\frac{c}{2 \lambda}\right)(x-c t)+\frac{\alpha A_{0}^{2}}{4 \lambda} \int^{x-c t}(\operatorname{sech} \xi)^{1 / 2} d \xi$,

where the latter integral can be rewritten via the substitution $\eta=\exp (\xi / 2)$ as

$\int^{x-c t}(\operatorname{sech} \xi)^{1 / 2} d \xi=2 \sqrt{2} \int^{\exp ((x-c t) / 2)} \frac{d \eta}{\sqrt{1+\eta^{4}}}$. 
This can then be expressed in terms of a standard elliptic integral using the representation

$$
\int_{z}^{\infty} \frac{d \eta}{\sqrt{1+\eta^{4}}}=\frac{1}{2} \mathrm{cn}^{-1}\left(\frac{z^{2}-1}{z^{2}+1}\right) .
$$

The relations (9), (12), (15), (16) determine an exact, localized, propagating pulse of the DNLS equation (1) subject to constraints (13), (14) on the physical parameters. This propagating envelope is illustrated in Figure 1.

In physical terms, this bright solitary pulse solution exists for the governing DNLS model with arbitrary dispersion, self-steepening, cubic and nonic nonlinearities, but in the absence of the seventh order nonlinearity and with the fifth order nonlinearity subject to the constraint expressed by the second equation of (14). Moreover, the nonic parameter $\sigma$ and the dispersion parameter $\lambda$ need to be of the same sign as indicated by the first equation of (13). However, by contrast, the relation (14) dictates that quintic parameter $v$ and dispersion parameter $\lambda$ must be of opposite signs. Thus, this particular localized pulse essentially exists as a result of a competition between quintic and nonic effects.

\section{A one-parameter class of dark solitary pulses}

The calculations of the previous section exploit the fact that the constants of motion $H, J$ vanish in the long wave limit. Here, we construct an exact periodic 
solution of the nonic DNLS equation (1) for which these invariants are nonvanishing. The transformation

$$
u=A_{0} \sqrt{\frac{1}{y^{2}+b}},
$$

is introduced into (10) whence it is seen that the structural equation

$$
\begin{aligned}
\left(\frac{d u}{d x}\right)^{2}= & -A_{0}^{2}-\left(3 b+k^{2}-2\right) u^{2}-\left[3 b^{2}+2\left(k^{2}-2\right) b+1-k^{2}\right] \frac{u^{4}}{A_{0}^{2}} \\
& -\left[b^{3}+\left(k^{2}-2\right) b^{2}+\left(1-k^{2}\right) b\right] \frac{u^{6}}{A_{0}^{4}},
\end{aligned}
$$

admits a solution

$$
u=\frac{A_{0}}{\sqrt{\operatorname{dn}^{2} x+b}} .
$$

Alignment of (6) and (17) now produces an exact solution of the nonic DNLS equation (1) with the constants of motion $J, H$, phase speed $c$, and angular frequency $\Omega$ given by

$$
\begin{aligned}
& J=\frac{A_{0}}{2}, \quad H=\frac{A_{0}}{2}\left(p-\frac{\mu}{\alpha}\right), \quad c=\frac{2 \lambda \mu}{\alpha}, \\
& \frac{\Omega}{\lambda}=\left(2 p-\frac{\mu}{\alpha}\right) \frac{\mu}{\alpha}-\frac{\alpha A_{0}}{4 \lambda}+\frac{3 b+k^{2}-2}{4} .
\end{aligned}
$$

The two parameters $A_{0}, b$ are associated with the peak and trough of the wave train, and, in the long wave limit, with the amplitude of the far field plane wave and the 
minimum intensity of the dark solitary pulse. The parameter modulus $k$ of the elliptic function determines the period of the wave pattern. The three parameters, $k$, $A_{0}$ and $b$ are related by the two constraints,

$$
3 b^{2}+2\left(k^{2}-2\right) b+1-k^{2}=\left(\frac{4 v}{3 \lambda}+\frac{\alpha^{2}}{4 \lambda^{2}}\right) A_{0}^{2},
$$

and

$$
b^{3}+\left(k^{2}-2\right) b^{2}+\left(1-k^{2}\right) b=\frac{4 \sigma A_{0}^{4}}{5 \lambda},
$$

Thus, this class of periodic wave patterns admits one free parameter. The modulus of the envelope is given by

$$
\sum=\frac{A_{0}}{\sqrt{\operatorname{dn}^{2}(x-c t)+b}} .
$$

Solitary wave limit

In the solitary wave limit ( $k \rightarrow 1)$, the long wave envelope is given by

$$
\sum=\frac{A_{0}}{Q}, \quad Q=\left[\operatorname{sech}^{2}(x-c t)+b\right]^{1 / 2}
$$

where the phase $\Theta$ is recovered from (8) through elliptic integrals of the second and third kinds, as in the bright solitary pulse case of the previous section. Physically, this long wave limit represents a dark pulse moving on a background (Figure 2). 


\section{Modulation instability}

One background plane wave field for the DNLS equation (1) is represented

by:

$A=B_{0} \exp \left[i\left(\mu B_{0}^{2}+v B_{0}^{4}+\sigma B_{0}^{8}\right) t\right]$

where $B_{0}$ is a real constant. Standard stability analysis is then performed by linearization around a small perturbation and seeking modal dependence in the form $\exp [\mathrm{i}(\rho x-s t)]$. This results in the dispersion relation

$\left(s-\alpha B_{0}^{2} \rho\right)^{2}=\lambda \rho^{2}\left(\lambda \rho^{2}-2 \mu B_{0}^{2}-4 v B_{0}^{4}-8 \sigma B_{0}^{8}\right)$

between $\rho$ and s. In the language of optical fibers, for the anomalous dispersion regime $(\lambda>0)$ and positive cubic nonlinearity $(\mu>0)$, if either fifth or ninth order nonlinearity is negative $(v<0$ or $\sigma<0)$, it is possible to have real $s$ for sufficiently large $B_{0}$. For the normal dispersion regime $(\lambda<0)$, there is further flexibility to adjust nonlinearity to obtain real $s$. Hence, importantly, modulation instability can be avoided in selected parameter regimes.

\section{Additional classes of nonic and higher order DNLS equations}

If alignment may be obtained with a nonlinear equation with a fourth order nonlinearity such as (10), then the traveling modes may be described by the classical elliptic functions. Here, a pair of illustrative classes of higher order DNLS equations is indicated where such reduction can be accomplished. 
Attention is restricted to derivative nonlinear Schrödinger equations of the

type:

$$
i A_{t}+A_{x x}+i W(\Sigma) A_{x}+V(\Sigma) A=0, \quad \Sigma=|A|^{2}
$$

in which case the nonlinear equation analogous to (6) is

$$
\begin{aligned}
& \dot{\Sigma}^{2}=4 \sum\left\{2 H+\left(p^{2}-\frac{\Omega}{\lambda}\right) \sum+2 p G-2 F\right\}-4\left[J+\left(p-\frac{c}{2 \lambda}\right) \sum+G\right]^{2}, \\
& W\left(\sum\right)=2 \lambda G^{\prime}\left(\sum\right), \quad V\left(\sum\right)=2 \lambda F^{\prime}(\Sigma) .
\end{aligned}
$$

The case with $W$ linear reduces to (1a). Particular illustrative extensions to quadratic and cubic $W$ are set down below:

- $W(\Sigma)=6 b \Sigma^{2}, V(\Sigma)=-5 b^{2} \Sigma^{4} / \lambda$ (b real) - In this case, the integration of the nonlinear equation may again be achieved in terms of elliptic functions. The corresponding nonic DNLS model is given by

$i A_{t}+\lambda A_{x x}+6 i b|A|^{4} A_{x}-5 b^{2}|A|^{8} A / \lambda=0$.

This equation admits traveling modes described by (9) which can be obtained analytically via (25) together with relations (7) and the analogue of (8) for the present generalized system, namely,

$$
\Theta=\int^{x-c t} \frac{J+\left(p-\frac{c}{2 \lambda}\right) \sum+G\left(\sum\right)}{\sum} d \xi .
$$


Indeed cubic and quintic nonlinearities can be incorporated in (26), and the squared wave packet amplitude $\Sigma$ can again be expressed in terms of elliptic functions.

- $W(\Sigma)=8 a \Sigma^{3}, V(\Sigma)=-7 a^{2} \Sigma^{6} / \lambda+4 a c \Sigma^{3} / \lambda$ (a real) - The corresponding DNLS model with seventh and thirteenth order nonlinearities is

$i A_{t}+\lambda A_{x x}+8 i a|A|^{6} A_{x}+\left(4 a c|A|^{6}-7 a^{2}|A|^{12}\right) A / \lambda=0$.

Cubic and quintic nonlinearities can be included in the governing equation (28) without any essential modification of the procedure leading to elliptic function solutions.

\section{Conclusions}

In this note, a procedure recently introduced in [18 - 20] involving the application of two integrals of motion in physical NLS models has been used to isolate, via elliptic integral representations, localized bright and dark pulses and particular periodic waves in a nonic DNLS equation and other models with polynomial nonlinearities. The localized structures characteristically exist due to a balance among higher order nonlinearities. The stability aspects of such privileged exact solutions typically require detailed individual analysis based on such balance aspects. It is remarked that exact solutions such as those presented here, carry over, mutatis mutandis to harmonic oscillator models with octic potential [29 - 31]. 
In general terms, the algorithmic procedure as employed here relies upon the existence in the nonlinear coupled systems of an integral of motion of the type

$$
J=\dot{\phi} \psi-\dot{\psi} \phi+\Phi\left(\sum\right)
$$

together with a Hamiltonian

$$
H=\frac{1}{2}\left[\dot{\phi}^{2}+\dot{\psi}^{2}\right]+\Psi\left(\sum\right)
$$

where $\sum=\phi^{2}+\psi^{2}$ is a squared wave amplitude. It is natural to seek the existence of overarching integrable Hamiltonian systems which incorporate both nonlinear dynamical systems admitting invariants of the type (29) and (30) as well as the well-established Ermakov-Ray-Reid systems (see [21 - 27]). Such comprehensive extended Ermakov-Ray-Reid systems have recently been constructed in [28].

\section{Acknowledgement}

Partial financial support for the present investigation has been provided by the University of Hong Kong Incentive Award Scheme. 


\section{References}

[1] Kh. I. Pushkarov, D. I. Pushkarov and I. V. Tomov, Self-action of light beams in nonlinear media: soliton solutions, Opt. Quant. Electr. 11 (1979) 471-478.

[2] N. N. Akhmediev and A. Ankiewicz, Solitons, Nonlinear Pulses and Beams, Chapman and Hall, London, 1997.

[3] Y. S. Kivshar and G. P. Agrawal, Optical Solitons: From Fibers to Photonic Crystals, Academic Press, San Diego, 2003.

[4] Z. Birnbaum and B. A. Malomed, Families of spatial solitons in a two-channel waveguide with the cubic-quintic nonlinearity, Physica D 237 (2008) 3252-3262.

[5] A. Peleg, Y. Chung, T. Dohnal and Q. M. Nguyen, Diverging probability density functions for flat-top solitary waves, Phys. Rev. E 80 (2009) 026602.

[6] J. Belmonte-Beitia and G. F. Calvo, Exact solutions for the quintic nonlinear Schrödinger equation with time and space modulated nonlinearities and potentials, Phys. Lett. A 373 (2009) 448-453.

[7] R. H. J. Grimshaw and S. Y. Annenkov, Water wave packets over variable depth, Stud. Appl. Math. 126 (2011) 409-427.

[8] E. Wamba, A. Mohamadou, T. B. Ekogo, J. Atangana and T. C. Kofane, Effects of three-body interactions in the parametric and modulational instabilities of Bose-Einstein condensates, Phys. Lett. A 375 (2011) 4288-4295. 
[9] A. Biswas, 1-Soliton solution of $1+2$ dimensional nonlinear Schrödinger's equation in power law media, Commun. Nonlinear Sci. Numer. Simulat. 14 (2009) 1830-1833.

[10] A. Biswas, Quasi-stationary optical solitons with power law nonlinearity, $J$. Phys. A. 36 (2003) 4581.

[11] L. H. Zhang and J. G. Si, New soliton and periodic solutions of $(1+2)-$ dimensional nonlinear Schrödinger equation with dual-power law nonlinearity, Commun. Nonlinear Sci. Numer. Simulat. 15 (2010) 2747-2754.

[12] V. I. Karpman and A. G. Shagalov, Solitons and their stability in high dispersive systems. I. Fourth-order nonlinear Schrödinger-type equations with power-law nonlinearities, Phys. Lett. A 228 (1997) 59-65.

[13] M. Saha, A. K. Sarma and A. Biswas, Dark optical solitons in power law media with time-dependent coefficients, Phys. Lett. A 373 (2009) 4438-4441.

[14] H. Liu, F. Yan and C. Xu, The bifurcation and exact travelling wave solutions of (1+2)-dimensional nonlinear Schrödinger equation with dualpower law nonlinearity, Nonlinear Dyn. 67 (2012) 465-473.

[15] M. Li, B. Tian, W. J. Liu, H. Q. Zhang and P. Wang, Dark and antidark solitons in the modified nonlinear Schrödinger equation accounting for the selfsteepening effect, Phys. Rev. E 81 (2010) 046606. 
[16] S. H. Han and Q. H. Park, Effect of self-steepening on optical solitons in a continuous wave background, Phys. Rev. E 83 (2011) 066601.

[17] M. Marklund, P. K. Shukla and L. Stenflo, Ultrashort solitons and kinetic effects in nonlinear metamaterials, Phys. Rev. E 73 (2006) 037601.

[18] C. Rogers, B. Malomed, J. H. Li and K. W. Chow, Propagating wave patterns in a derivative nonlinear Schrödinger system with quintic nonlinearity, J. Phys. Soc. Jpn. 81 (2012) 094005.

[19] C. Rogers and K. W. Chow, Localized pulses for the quintic derivative nonlinear Schrödinger equation on a continuous-wave background, Phys. Rev. E 86 (2012) 037601.

[20] C. Rogers, B. Malomed and K. W. Chow, Invariants in a resonant nonlinear Schrödinger model, J. Phys. A-Math. Theor. 45 (2012) 155205.

[21] J. R. Ray, Nonlinear superposition law for generalised Ermakov systems, Phys. Lett. A 78 (1980) 4-6.

[22] J. L. Reid and J. R. Ray, Ermakov systems, nonlinear superposition and solution of nonlinear equations of motion, J. Math. Phys. 21 (1980) 1583-1587.

[23] C. Rogers and W. K. Schief, Multi-component Ermakov systems: structure and linearization, J. Math. Anal. Appl. 198 (1986) 194-220.

[24] C. Rogers , C. Hoenselaers and J. R. Ray, On 2+1-dimensional Ermakov systems, J. Phys. A. Math. Gen. 26 (1993) 2625-2633. 
[25] W. K. Schief, C. Rogers and A. Bassom, Ermakov systems with arbitrary order and dimension, Structure and linearization, J. Phys. A: Math. Gen. 29 (1996) 903-911.

[26] C. Rogers, B. Malomed, K. W. Chow and H. An, Ermakov-Ray-Reid systems in nonlinear optics, J. Phys. A. Math. \& Theor. 43 (2010) 455214.

[27] C. Rogers, B. Malomed and H. An, Ermakov-Ray-Reid reductions of variational approximations in nonlinear optics, Stud. Appl. Math. 129 (2012) 389413.

[28] C. Rogers, Integrable substructure in a Korteweg capillarity model. Generalised Ermakov-Ray-Reid systems, under submission.

[29] M. Jafarpour and T. Tahamtan, Octic anharmonic oscillators: Perturbed coherent states and the classical limit, Int. J. Theor. Phys. 48 (2009) 487-496.

[30] D. Agboola and Y. Z. Zhang, Exact solutions of the Schrödinger equation with spherically symmetric octic potential, Mod. Phys. Lett. A 27 (2012) 1250112. [31] S. Dhatt and K. Bhattacharyya, Asymptotic response of observables from divergent weak-coupling expansions: A fractional-calculus-assisted Padé technique, Phys. Rev. E 86 (2012) 026711. 


\section{Figures Captions}

(1) (Color online) Wave intensity $|A|^{2}$ versus $x$ and $t$ for a bright pulse of Eq. (1), $\lambda$

$=1, \mu=1, \alpha=1, v=-3 / 16, c=2, \delta=0, \sigma=1, A_{0}=(5 / 16)^{1 / 8}$.

(2) (Color online) Wave intensity $|A|^{2}$ versus $x$ and $t$ for a dark pulse of Eq. (1), $\lambda=$

$1, \mu=1, \alpha=1, v=1, c=2, \delta=0, \sigma=-1, b=0.863, A_{0}=0.599$. 


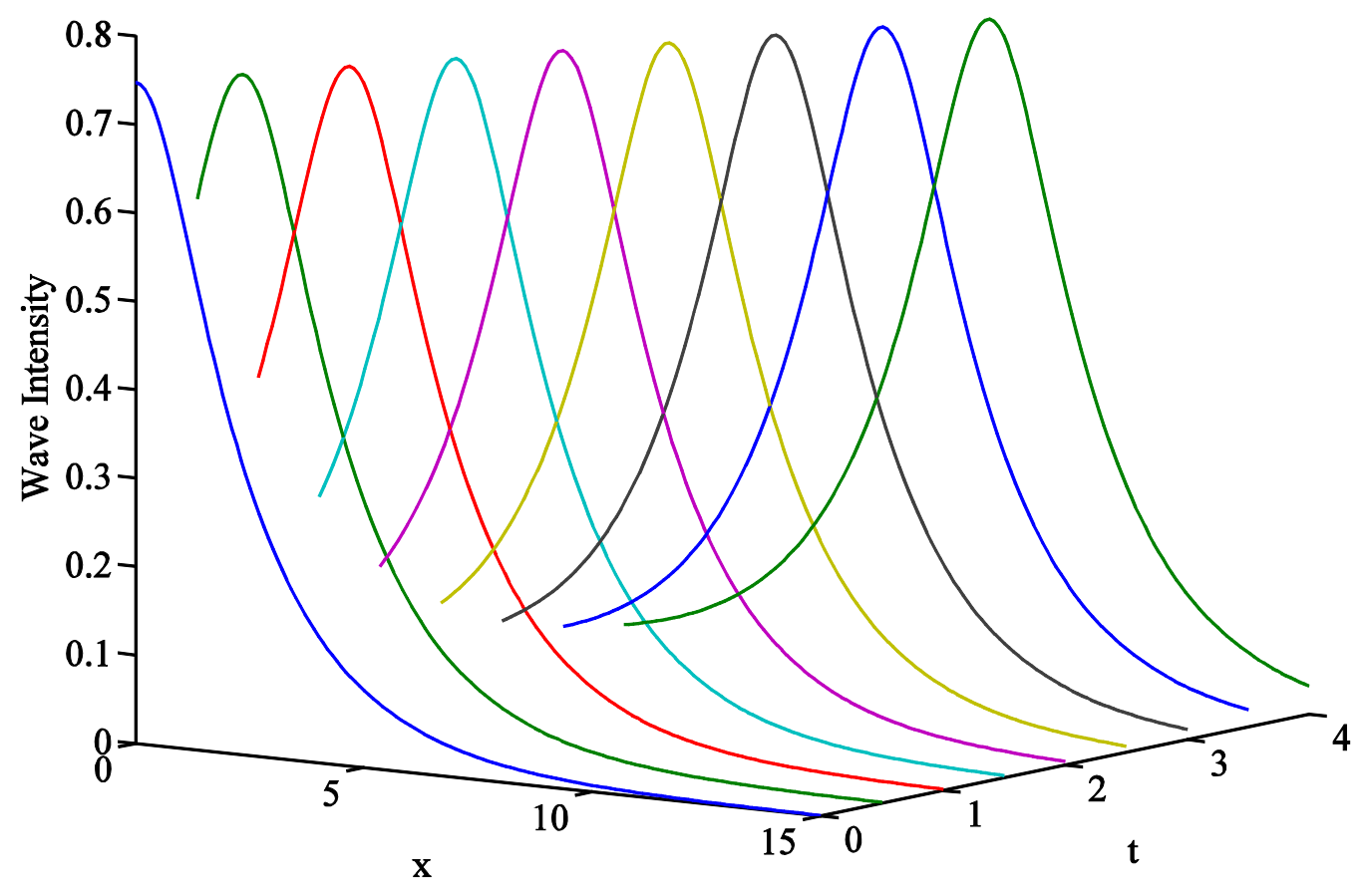

Figure 1 


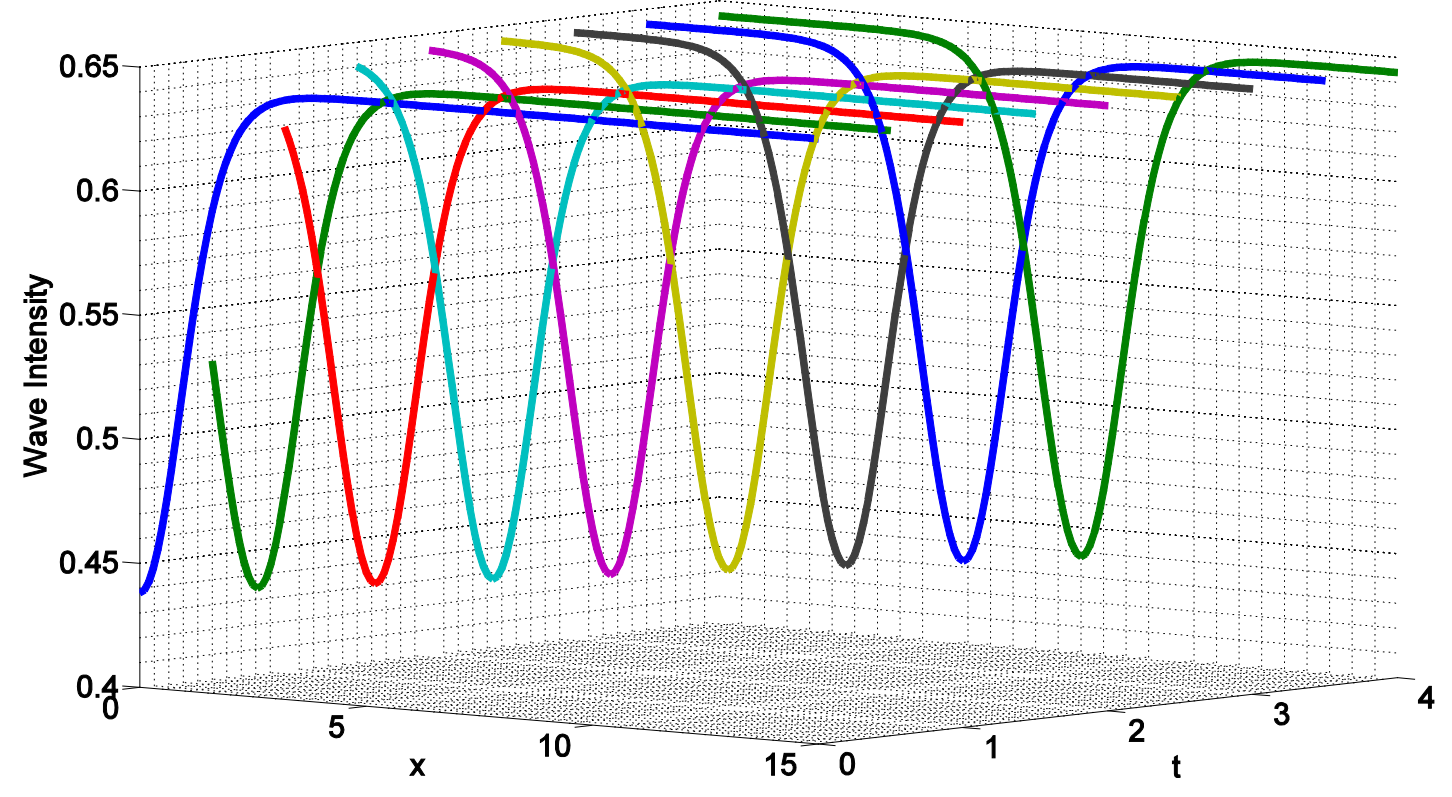

Figure 2 